\title{
sistemas integrados de información técnica en fábricas de cemento
}

\author{
J. REETZ \\ IBM Alemania (Stuttgart)
}

\section{INTRODUCCION}

Cuando en la actualidad es tratado en el campo industrial el concepto "sistemas de información", se hace generalmente con miras a la cantidad de datos que deben ser preparados para la comercialización. En numerosas empresas es cada vez mayor la tendencia a la rápida información del rendimiento del negocio; en ellas fueron introducidos primeramente los sistemas de información para la Dirección con sus conceptos básicos. En la industria del cemento no está la justificación, para la aplicación de este sistema, en la rapidez de la información sobre el desarrollo del funcionamiento, sino más bien en el volumen de dicha información. Muchas fábricas o sociedades están provistas, además, con instalaciones de elaboración de datos para fines comerciales que, en su capacidad y en el grado de condensación de la información, satisfacen la organización de los datos allí elaborados; pero no comprenden, sin embargo, valores particulares del campo técnico, sino que son primeramente recopilados manualmente y almacenados del mismo modo. Las tareas de esta clase ocupan, sin embargo, una parte considerable del personal disponible, que debe considerar y evaluar las columnas de datos de medida aparecidas en la instalación. La importancia de esta recopilación tampoco es muy elevada, es decir, no son posibles, sin inconvenientes, comprobaciones sobre el comportamiento de la instalación y juicios sobre la exactitud de determinados equipos. Dichas desventajas del tratamiento convencional han originado el deseo, en el caso de los técnicos avanzados, de evaluar los datos disponibles, con la ayuda de instalaciones electrónicas de elaboración de datos, siguiendo diversos criterios.

Naturalmente, no es posible crear todo un sistema completo de información de una empresa con una sola calculadora de procesos. Por lo tanto, sólo serán estudiadas secciones parciales que puedan ser controladas con gastos relativamente reducidos.

\section{DESARROLLO}

Algunas fábricas de cemento disponen ya de calculadoras de procesos para la vigilancia y regulación de la instalación. Muchos de esos sistemas abarcan el total de la fá- 
brica, de modo que todos los datos pueden ser leídos en la memoria de la calculadora de procesos, en tanto que sean obtenibles mediante la técnica de medida. Con ello se ha resuelto en gran parte el problema para estas fábricas. El procesamiento de datos se utiliza generalmente para regulación de instalaciones y no, en cambio, para análisis estáticos de las instalaciones existentes.

Como muestra la experiencia, las calculadoras de procesos aplicadas están enteramente en situación de resolver los problemas tecnológicos de la regulación de instalaciones. Por consiguiente, en este campo no son de esperar ya mejoras sorprendentes para los procedimientos de producción hoy conocidos. Sin embargo, son necesarias mejoras en el campo de la técnica de medidas, como, por ejemplo, aumento de la precisión y exactitud de los instrumentos. Dichos progresos necesitan su tiempo, independientemente de la elaboración de la información suministrada por ellos.

Para la regulación de las instalaciones de producción en las fábricas de cemento no es imprescindible utilizar calculadoras rápidas, ya que son suficientes sistemas de exploración con un ciclo de aproximadamente 10 segundos. Por otra parte, las calculadoras de procesos de serie disponibles actualmente son tan rápidas que les sobra capacidad de cálculo y acumulación para otras tareas. Con ello satisfacen el deseo de la fábrica al permitir más investigaciones sobre el comportamiento de las instalaciones y un mejor conocimiento de los procesos. Una de las aplicaciones posibles es la integración y evaluación de las informaciones de datos de medida leídos en el calculador de procesos. Una condición para conseguir uno de tales sistemas técnicos de información es la programación de la calculadora en FORTRAN o en otro lenguaje de calidad, y la posibilidad de dejar marchar estos programas de sistemas de tiempo compartido junto con los programas de regulación necesarios. Actualmente es aún desconocida la parte de costos de cálculo que se pueden justificar con tal sistema de información. El artículo debe mostrar la posibilidad y el volumen de un sistema técnico de información.

\section{ESTRUCTURA DEL SISTEMA}

\subsection{RESUMEN DEL SISTEMA}

Un sistema de información consta, esencialmente, de las siguientes partes:

Banco de datos.

Programas para la acumulación de información.

Programas para la evaluación de las informaciones.

Vigilancia y regulación de las instalaciones.

En este caso se ha supuesto un calculador de procesos como soporte del sistema de información.

\subsection{BANCO DE DATOS}

Es la parte más importante de un sistema de información y también su centro funcional. Las informaciones pueden ser almacenadas según dos puntos de vista distintos:

clasificación de los datos durante su conocimiento;

clasificación de los datos en la evaluación. 
Si se realiza un sistema de información sobre una instalación comercial de cálculo, las consideraciones de tiempo para la salida de datos sólo tienen un valor secundario. Por lo tanto, se puede ejecutar, consecuentemente, uno de los principios antes indicados. Sin embargo, si el banco de datos está en una calculadora de procesos, es decir, que deban servirse también de él programas de control y regulación de la instalación, no es posible la sola clasificación de datos, sino que se necesita también su evaluación. Por este motivo, deben ser recopilados en bancos de datos auxiliares los datos necesarios para los programas de vigilancia y regulación, con objeto de posibilitar un rápido aprovechamiento de los programas de procesos. En general se debe partir del supuesto de que, por lo menos, deben estar disponibles continuamente en la memoria los valores de medida de todas las instalaciones en unidades físicas. Allí están asimismo disponibles los datos primarios, es decir, los valores de salida del convertidor analógico-digital. Las prescripciones de tratamiento de estos datos deben, sin embargo, encontrarse en el acumulador externo (placa o tambor), ya que no son necesarios en cortos intervalos de tiempo. A dichas prescripciones de tratamiento pertenecen:

tipo de los filtros y curvas de tarado;

constantes del filtro;

parámetros de las curvas de tarado;

límites de servicio y regulación;

indicador del estado de los aparatos de medida;

textos para los avisos de alarma de las magnitudes de medida individuales.

Naturalmente deben mantenerse al día estos ficheros del banco de datos.

Por otra parte, el banco principal de datos puede ser dividido, según el tiempo de utilización permitido, en:

modificaciones en intervalos de minutos;

modificaciones en intervalos de algunas horas, hasta 1 día.

Con ello es posible mantener los datos raramente utilizados en un acumulador externo intercambiable, que sólo en caso de necesidad, por ejemplo por modificación o evaluación de los datos, está unido con el sistema de cálculo.

Las cartotecas o ficheros se han de organizar de forma tal que determinados programas de inscripción se agrupen según el ciclo de trabajo. Esto significa que todas las constantes que pertenecen a la conversión de magnitudes de medida y vigilancia son acumuladas del mismo modo para cada lugar de medida, en un orden determinado; es decir, que cada punto de medida tiene asociado un grupo de datos de longitud determinada y constante.

Por ejemplo, para datos históricos puede conseguirse una cartoteca similar de dos dimensiones. En este caso, el número de grupos de datos, depende solamente del intervalo de tiempo pasado.

Esta clase de organización de datos tiene, naturalmente, la desventaja de que el espacio disponible en el acumulador no se puede aprovechar óptimamente, ya que no son 
necesarios para cada magnitud de medida el número máximo de parámetros de cálculo previstos. Las cartotecas individuales de la instalación aquí considerada se tratan en la sección 4.

El aprovechamiento de las cartotecas se ha organizado principalmente de manera que los programas de inscripción y evaluación reciben, a través de tablas con indicadores, determinadas informaciones básicas sobre la estructura de las cartotecas a tratar. Sin embargo, por causas del aprovechamiento del acumulador no es muy adecuado el leer todas las informaciones de estructura en estas tablas de mando, sino que son montadas en los programas de inscripción y de evaluación. La solución intermedia así aparecida frente a un sistema puro de interpretación parece ser un excelente compromiso dado el estado actual del desarrollo de los lenguajes de sistemas de información, en relación con la exigencia de acumulador y con los gastos de programación. Ello representa, en el ejemplo de la cartoteca de valores de medida, que en la posición del acumulador de los parámetros de cálculo en el grupo de datos se supone un punto de medida desde el programa. Esta solución se habrá superado en algunos años cuando estén disponibles los lenguajes de sistema, orientados por bloques, que trabajan como intérpretes.

\subsection{PROGRAMACION}

Los programas para escribir el banco de datos se han de dividir en dos grupos:

Carga, por primera vez, de los valores consigna y de los valores variables.

Entretenimiento continuo de las constantes y marcha intermitente de las variables.

Las fuentes de datos se pueden dividir en cuatro grupos:

Los valores de medida del proceso son filtrados a través de programas de compresión controlados en sus correspondientes valores límites y, según su aplicación, promediados e integrados. Paralelamente a la inscripción de esos valores de medida en el banco de datos tiene lugar su evaluación en programas para la regulación de las instalaciones.

El personal de servicio puede introducir a través del pupitre de mando complementario, en el puesto de control de medidas, las modificaciones necesarias de las constantes para el servicio de esos programas de regulación. De este modo es posible modificar, por ejemplo, los valores complementarios de aparatos de medida fuera de servicio, o el tipo de funcionamiento deseado. También pueden ser solicitados desde el pupitre los programas que no son efectuados en función del tiempo. Naturalmente los datos aquí sugeridos se han de examinar, incluso bajo determinados criterios de seguridad, antes de ser aceptados en los ficheros de tarjetas. Operando de esta manera, el ingeniero de procedimientos, sólo tiene la posibilidad, por ejemplo, de intervenir mediante un conmutador para modificar determinados valores de medida de los circuitos de regulación o para variar el comportamiento del tiempo de regulación.

En la puesta en marcha del sistema deben ser rellenados los ficheros de tarjetas, siendo la forma más adecuada para efectuarlo el empleo de máquinas perforadoras con sus correspondientes lectoras de tarjetas. Lo mejor es prever programas propios para la primera carga de datos, ya que $\epsilon$ n el examen intensivo de las 
tarjetas a leer no hay limitaciones en cuanto al tiempo de ejecución, debido a que el calculador de procesos aún no manda la instalación. Por el contrario, los programas deben tomar en consideración esa limitación de tiempo, los cuales, durante el servicio de regulación, leen nuevos valores en el almacén de datos. Otra misión de los programas de lectura consiste en la toma de datos estáticos que fueron perforados anteriormente en un momento cualquiera y que posteriormente han de ser evaluados en un desarrollo de programa separado.

No siempre es posible leer los nuevos valores directamente para el banco de datos a través de la unidad adicional de tarjetas. Para este fin, deben ser comunicadas directamente al calculador de procesos, a través de aparatos de elaboración de datos a distancia, las informaciones aparecidas en el lugar alejado. A ellas pertenecen, tanto los valores de medida de instalaciones bastante alejadas cuya conexión directa no es aconsejable por el elevado coste de los cables, como las constantes que, por ejemplo, deben ser transmitidas desde una calculadora central de la empresa (precios de producto, exigencias del mercado, especificaciones de productos). Los programas aquí necesarios de inscripción procuran el funcionamiento en tampón de los avisos, que aparecen secuencialmente y mandan, igualmente, a las instalaciones de transmisión.

\subsection{FUNCIONES DE EVALUACION}

Los programas en correspondencia con el banco de datos pueden ser divididos en tres niveles de jerarquía:

acumulación de las informaciones;

análisis del servicio;

control y optimación del servicio.

Sobre dichos tres niveles se pueden subdividir otra vez las funciones según sus campos de actividad en la fábrica:

servicio técnico;

entretenimiento de las instalaciones;

cálculo de costos.

Los sectores individuales de esa matriz serán tratados en el siguiente capítulo.

\section{APLICACIONES}

En este capítulo serán estudiados, con más detalle, los campos de la matriz antes indicada, en el sentido de sus posibilidades de aplicación a las fábricas de cemento.

\subsection{SERVICIO TECNICO}

Este campo de función comprende, sobre todo, asuntos referentes al control y regu- 
lación de las instalaciones de servicio; sin embargo, incluye también el mando superior del proceso de producción y la ayuda del calculador de procesos para trabajos de análisis en el laboratorio de la fábrica.

\subsubsection{Acumulación de las informaciones}

La acumulación de las informaciones variables (datos de procesos) se ocupa de los programas de comprensión de datos para las instalaciones de la fábrica. Estos programas exploran los puntos de medida conectados y examinan el valor de las medidas en cuanto a exactitud y peligro para la instalación. Tras la inscripción de dichas informaciones variables de la instalación en el almacén de valores de medida, éstas pueden ser resumidas en informaciones sobre el transcurso de la producción, es decir, en controles de turno y jornada de trabajo. Otras informaciones, como, por ejemplo, las de fin de mes, deben aprovechar datos adicionales que no se pueden consultar directamente al calculador de procesos, sino que son introducidos al mismo, por la contabilidad de la fábrica, en forma de constantes inscritas en tarjetas perforadas. Su proporción es no obstante muy reducida (por debajo del $10 \%$ ) frente a las magnitudes de servicio medibles directamente.

El fichero de los valores de medida permite, en este nivel del desarrollo del sistema, la regulación directa de las instalaciones conectadas, en tanto sea conocido su comportamiento temporal y estén disponibles las magnitudes de medida y ajuste necesarias; debido a que en los acumuladores externos pueden mantenerse, durante largo tiempo, los valores de medida, es también posible el análisis separado, y seleccionar los valores de medida característicos para las instalaciones correspondientes.

Al campo del mando de las instalaciones pertenece también la evaluación directa de los aparatos de análisis, por ejemplo, un analizador de fluorescencia Röntgen. Su misión más importante es, en general, el análisis del crudo sobre el que se hace la regulación de la mezcla. Si para este fin es aplicado un aparato de perlas o tabletas, se pueden solucionar, sin dificultades, los análisis normales para cantera, crudo, clínker y cemento, y los correspondientes problemas de evaluación. El trabajo del personal del laboratorio se puede limitar a la determinación de la cal libre en el clínker, y a la finura de molido.

Un balance de cada instalación, que es normal efectuar en otras industrias, no es posible hacerlo, desgraciadamente, en la fábrica de cemento, ya que, por ejemplo, para el importante balance térmico no se han proyectado aún los aparatos de medida necesarios o no tienen la suficiente exactitud o precisión y libertad de entretenimiento. Por el contrario, es posible la disposición de balances de inventario para los silos de material; estos son los silos de alimentación de los molinos de bolas, los silos de polvo y los silos de cemento entre molinos y envasado. El mayor almacén de material en la fábrica de cemento es, de todos modos, el de clínker, cuyo nivel sólo se puede determinar con exactitud para pequeñas cargas.

Las indicaciones de existencias se determinan con la ayuda de un factor crudo-clínker cuyo valor no depende tanto de los procesos físicos como de las consideraciones técnicas de producción.

Con el auxilio del fichero de valores de medida puede, sin embargo, atacarse una serie de tareas en el campo de la tecnología de procedimientos, que parecen irrealizables sin el calculador de procesos. A ello corresponden: 
- La aplicación de métodos estadísticos sobre la variación temporal de las magnitudes de medida. Mediante cálculos de autocorrelación o de correlación cruzada es posible estudiar, tanto procesos dinámicos como estacionarios de los valores de medida de instalaciones particulares. Estos conocimientos pueden utilizarse entonces para mejorar los programas de regulación antes indicados.

- La existencia de los valores de medida en su correspondiente fichero, facilita y hace posible la planificación y ejecución de interesantes series de experiencias. Junto con el banco de datos definitivamente estructurado pueden ser introducidos conjuntos de datos adicionales para dichas investigaciones, que han de ser inscritos y más tarde evaluados mediante nuevos programas. De todos modos, dichos programas sólo pueden ser tomados por calculadoras de procesos con programas de mando correspondientes para el servicio de tiempo compartido.

\subsubsection{Análisis y planificación}

Los métodos antes indicados del cálculo de correlación pueden satisfacer, en una parte considerable, la misión del análisis de sistemas para instalaciones elegidas. La evaluación de los valores de medida acumulados según otros criterios de la técnica de regulación es, sin embargo, posible sin dificultades y sólo exige la creación de los programas adecuados. El gasto, en cuanto a su desarrollo en relación a la posible utilidad, es soportable únicamente cuando en este nivel de la condensación de datos pueden utilizarse lenguajes de programación como, por ejemplo, el FORTRAN.

Partiendo de un análisis puramente estadístico del comportamiento de las instalaciones pueden desarrollarse modelos, que describen las instalaciones de forma puramente estadística o interpretan sus propiedades fisicoquímicas. Paralelamente a dichos trabajos de desarrollo tienen lugar las evaluaciones normales de los programas de regulación ya instalados.

Sólo la elaboración de las tareas -antes descritas- permite ahora optimizar dinámicamente los programas de regulación de las instalaciones; con esto, no está unida sin embargo aún ninguna optimación del servicio, es decir, los valores nominales previstos para el proceso técnico.

Independientemente de dichos trabajos teóricos, pueden marchar los mandos de producción en la primera fase de la instalación del sistema. Fundamentalmente, este control es también una regulación superior del flujo del material; sin embargo, se considera un gran número de condiciones auxiliares que son dadas por la dirección del servicio o por la capacidad máxima de producción de cada circuito.

En muchas fábricas se reciben envíos de materiales por otras firmas, pero son controlados por la dirección de distribución de la fábrica. Las posibilidades de aplicación de cada medio de transporte, según el criterio de los costos más reducidos, o bien del camino total mínimo, exigen siempre la introducción de las magnitudes de medida necesarias a través de pulsadores o tarjetas perforadas, por la imposibilidad de estar contenidas directamente en el programa.

Dichas misiones se han efectuado hasta ahora satisfactoriamente de forma manual y no necesitan imprescindiblemente la utilización de la calculadora. 


\subsubsection{Marcha del servicio}

Las misiones de estos campos de trabajo exigen más informaciones de las que pueden estar contenidas directamente en los conjuntos de datos tratados hasta ahora. Para este fin debe introducirse, para los siguientes programas de evaluación, una asociación con los conjuntos de datos del cálculo de costos y de expedición. Sólo así es posible, por ejemplo, el mando de la producción de la fábrica según especificación de productos y calidades para largos intervalos de tiempo. Naturalmente, ello tiene lugar actualmente con buenos resultados en toda fábrica, puesto que las condiciones del mercado para la industria del cemento son relativamente simples y fácilmente visibles. El mismo argumento es válido para la planificación de las paradas de las instalaciones para su mantenimiento ; planificación efectuada tradicionalmente en los meses de invierno, que es precisamente cuando bajan las ventas.

Las dos misiones - antes indicadas- de dirección del servicio no tiene objeto efectuarlas mediante la calculadora, pues el sistema de información, según todas las posibilidades, no facilitará ningún resultado diferente a la de los métodos de planificación actual.

No obstante, otro campo del control del servicio exige gran atención: la optimación de la marcha de las instalaciones. Los valores nominales hoy ajustados o exigidos para las magnitudes de medida características de las instalaciones, no son óptimos en gran número de los casos. Algunas fábricas han efectuado series de experiencias sobre la dependencia de las perturbaciones en el servicio, de los parámetros variables a voluntad; sin embargo, no es suficiente para las exigencias este relativamente pequeño número de valores, como para permitir decisiones justificadas. Precisamente en este sector se presenta, como magnitud inconmensurable, la diferente experiencia individual del jefe de servicio. La integración de todas las medidas permite ahora acumular experiencias en el tiempo más corto, con lo que se pueden facilitar algunas funciones de la dirección del servicio.

Incluso cuando se materializa esta experiencia, es decir, una vez se ha programado como proceso de regulación, considerando todas las restantes condiciones marginales, en ningún caso, traería como consecuencia, que se redujese la importancia del director de servicio. El debiera, por el contrario, agradecer que fueran efectuadas sin su concurso decisiones casi rutinarias. Aparte de ello sabe que, durante su ausencia, son conducidas las instalaciones según sus criterios.

\subsection{MANTENIMIENTO DE LAS INSTALACIONES}

Para este sector del sistema de información son necesarios otros conjuntos de datos:

- Instalaciones y grupos de datos sobre:

- gastos de reparaciones para las averías posibles;

- necesidades de personal y costos de una reparación;

- costos acumulados dentro de un año.

- Almacén de piezas de repuesto con conjuntos de datos para:

- descripción de las piezas de repuesto, incluido costos:

- valor experimental de la frecuencia de reposición; 
- cantidad mínima en existencia;

- economía en los pedidos;

- ordenación con los equipos en la fábrica.

- Servicio de instalaciones:

- tipos de avería y duración;

- causas de las averías;

- tiempo de marcha de las instalaciones y grupos;

- clase e intervalo de tiempo entre inspecciones;

- decisión sobre si se han de mantener en marcha las instalaciones hasta que fallan o si se han de revisar periódicamente.

- Instalación de cálculo:

- indicaciones a través de instrumentos de medida, cableado, métodos de elaboración en la calculadora;

- datos de la programación, como, por ejemplo, parámetros de bucles de regulación, lenguajes, intervalos entre llamadas, etc.

\subsubsection{Acumulación de las informaciones}

Planificación del personal

Si la fábrica trabaja según el principio del entretenimiento preventivo, es posible, sin inconveniente alguno, planificar para largo tiempo la distribución de los trabajos y la cualificación del personal necesario. Desgraciadamente no es posible alcanzar en la industria del cemento una dedicación única en el trabajo para el personal de entretenimiento, como consecuencia de su carácter de temporada. El mayor número de exigencias se refieren a reparaciones inesperadas y a que no hay tiempo para preparar, con la ayuda de la calculadora, las informaciones de planificación e instrucciones para los trabajos, pues previamente ésta tiene que recibir todas las constantes necesarias para la realización de los mismos. Por lo tanto, sólo es posible para este sector coleccionar datos históricos y extraer informes resumidos sobre el mantenimiento de las instalaciones y el gasto del personal.

\section{Almacén de piezas de repuesto}

El almacén de piezas de repuesto en las fábricas de cemento está estructurado fundamentalmente de otro modo que, por ejemplo, en los talleres de acabado de la construcción de máquinas. Sus características sobresalientes son:

- menos movimientos;

- reducidas cantidades (a menudo 1) ya que en las fábricas de cemento sólo hay contadas secciones de igual actividad. 
Por ello no es posible una predicción del movimiento de almacén, ya que las estadísticas necesarias para ello presentan grandes dispersiones. Por el contrario, el sistema de información puede tomar a su cargo el control del almacén con pocos gastos y extraer informes sobre los costos de mantenimiento. Los portadores de esas informaciones son o bien tarjetas perforadas, que son efectuadas para cada movimiento del almacén; o bien acumuladores externos de datos, que son inscritos directamente a través de pulsadores con dos símbolos de exigencias ( . $^{\circ}$ de pieza, cantidad, precios). La reducida frecuencia de movimiento permite inscribir de nuevo, en intervalos de aproximadamente 1 día, el conjunto de datos correspondiente.

\section{Servicio de instalaciones}

A las funciones de evaluación referentes a las instalaciones pertenecen:

- Informes sobre las averías y el tiempo de marcha de las instalaciones.

- Informes sobre el número y la magnitud de las inspecciones periódicas.

- Panorama de los costos de entretenimiento de cada instalación o bien de cada conjunto. Estos costos de entretenimiento son extraídos partiendo del conjunto de datos de reparaciones referidos al personal y del conjunto de datos del almacén de piezas de repuesto.

- A este sector pertenecen también informes evaluatorios sobre los costos de la pérdida de producción motivados por inopinados fallos en secciones. Estos sirven como base para decidir si esas instalaciones deben repararse con más frecuencia.

\section{Conexión del CAlCulador de PRocesos}

Estas conexiones están representadas, de la forma usual, en dibujos de circuitos de medida y de conexionado. Ello significa que las informaciones que pertenecen a un único circuito de medida deben buscarse conjuntamente en muchos dibujos. Por otro lado, son utilizadas, en los programas de cálculo, muchas de las indicaciones allí contenidas, de forma que es adecuado el que estén contenidas, en un conjunto de datos, todas las informaciones que pertenecen a la periferia de proceso del calculador. Este conjunto de datos contiene, para cada circuito de medida o de ajuste, las siguientes indicaciones importantes:

- tipo y número del captador de medida;

- tipo y número del transportador de señales;

- clase de alimentación de corriente;

- detalles del camino de la señal, como, por ejemplo:

- regletas de bornas y número de las bornas;

- tipo de cables y número de venas.

Junto con dichos datos para la transmisión de señales, se deben indicar otras constantes para la agrupación y para los programas de regulación. Las constantes aquí indicadas deben estar disponibles en todo momento para los programas correspondientes; mientras que los datos para la transmisión pura de señales sólo se han de conectar, al sistema de calculadoras, en el caso de modificaciones que se presente con relativa poca frecuencia. 


\section{Programas}

Indicaciones semejantes se presentan para la programación de la calculadora. Por ejemplo en cada programa se han de indicar:

- clave;

$$
\text { 江泽 }
$$

- tipo del programa (proceso o no proceso);

- intervalo entre consultas;

- condiciones de consulta;

- programador ;

- fecha de la última modificación;

- necesidades de espacio y residencia.

Las dos colecciones de datos antes desarrolladas pueden ser evaluadas según los distintos puntos de vista, es decir, facilitar informes. Estos tipos de órdenes están clasificados de diversos modos según el trabajo, o bien el sector de interés de las consultas:

- técnicos de medida;

- técnicos de regulación;

- programadores ;

- personal de reparaciones;

- director de proyectos.

Los programas de evaluación están contenidos simultáneamente, con los conjuntos de datos sobre acumuladores externos, ya que sólo son necesarios, en el sistema de calculadoras, en caso de modificaciones o de tareas de información.

\subsubsection{Análisis}

Una evaluación que se ha convertido entretanto en clásica para datos de almacén, es la predicción de las necesidades para poder efectuar los pedidos con la suficiente antelación. La estructura de los almacenes de piezas de repuesto normales en la industria del cemento, no permite ninguna predicción segura sobre las necesidades como consecuencia de la gran dispersión de los intervalos de tiempo en necesitar piezas iguales, de modo que se elimina esa posibilidad de evaluación de los datos de almacén.

Pero pueden utilizarse, preferentemente, para comparar la necesidad de piezas de repuesto, según los diversos puntos de vista. Si han sido suministradas, por distintos fabricantes, instalaciones con la misma función, esta evaluación permite también la comparación de la calidad de los grupos utilizados. La opinión sólo puede tener un valor limitado, pues las instalaciones de una fábrica sólo provienen de pocos fabricantes.

\subsubsection{Planificación y control}

Las experiencias conseguidas hasta ahora muestran que el mantenimiento preventivo es posible sólo para un determinado tipo de aparatos e instalaciones. Para todos los 
elementos restantes, es maycr el costo para una revisión preventiva que el de reparación en caso de imprevistos. De ello resulta que no es posible la planificación del mantenimiento de las instalaciones para todos los grupos.

En este sector deben conseguirse, por lo tanto, aún muchos más valores de experiencia en representación adecuada para el cálculo, a fin de poder decidir sobre la constitución de la planificación del entretenimiento en el sistema de información.

La cantidad, aún abarcable, de grupos de producción, tampoco hace necesario que, por ejemplo, el sistema de información presente los informes, en el tiempo real, sobre los trabajos de entretenimiento. El costo para la creación de esta parte del sistema sólo debiera ser aconsejable para fábricas con más de 4 o 5 líneas de producción.

\subsubsection{Ventajas del sistema de entretenimiento}

Las ventajas de tipo normal dadas se anulan en gran parte para la industria del cemento. De este modo, el costo para la presentación de los datos de entretenimiento, como, por ejemplo:

- cualificación del personal,

- exigencias de entretenimiento,

- tiempo de observación permitido,

no guarda ninguna relación correcta en el posible aprovechamiento, es decir, para con el aprovechamiento uniforme del personal y de los restantes servicios de entretenimiento. Debido a que tampoco es de esperar ninguna reducción importante en las existencias del almacén de piezas de repuesto, queda, como casi única ventaja del sistema de entretenimiento, la mejor posibilidad de juicio de la calidad de cada instalación e instrumentos.

\subsection{CALCULO DE COSTOS}

En este sector sólo deben ser investigadas dos misiones - cuyas informaciones pueden ser tomadas en gran parte directamente de la calculadora de procesos-, que son la expedición y la distribución de costos.

\subsubsection{Recopilación de información}

Expedición:

Para la expedición son fáciles de conectar al calculador las fuentes importantes de información:

- existencias y clases, en los silos de cemento;

- cantidad y clase de los pedidos satisfechos;

- pesos de entrada y salida de los medios de transporte (camiones, vagones).

En el fichero de datos están contenidos además:

- número del cliente y deseos en cuanto a calidad; 
- condiciones de facturación;

- tiempo de espera permitido.

En general no pertenecen a la fábrica los medios de transporte, de modo que no parece interesante ocuparse de la optimación del trayecto; sin embargo, puede cobrar importancia cuando los plazos de entrega permitidos pueden ser sobrepasados en la planificación manual de la aplicación del medio de transporte. Con la toma de este trabajo en el sistema de información pueden descargarse los medios de transporte unidos por contrato. Para ello es necesario la introducción de los datos mediante pulsadores con objeto de comunicar a la calculadora qué camiones están en camino para cada cliente, así como un cálculo de los tiempos de viaje y de descarga y otros impedimentos que dificultan el plazo de entrega. El sistema puede indicar, consultándolo, el suministro más rápido posible para un cliente conociendo previamente el lugar de entrega, de forma que resulte del modo más rápido o bien el más económico con los medios de transporte disponibles.

La cartoteca de expediciones se ha de completar, aun para cada proceso de carga, mediante los siguientes datos:

- número característico del medio de transporte;

- número característico de la empresa de transportes.

La clase del medio de transporte viene fijado por los coches agregados correspondientes y por el código del teclado, a través del cual se dan las correspondientes indicaciones sobre los medios de transporte. Con dichas indicaciones está completo el conjunto de datos de expedición y, según ellos, están en disposición los programas de evaluación, los de rellenar los avisos de expedición y los de transmitir las bases para la facturación al departamento de contabilidad. Si es aplicada para este fin una instalación propia, por ejemplo en la administración del grupo industrial, puede transmitir los datos la calculadora de procesos mediante teletipos.

\section{Costos de producción}

En relación con el cálculo de costos para cada departamento ya están contenidas las siguientes indicaciones en el banco de datos:

- necesidad de piezas de repuesto y costos correspondientes;

- tiempo de marcha y de avería de las instalaciones;

- cantidades de producción, y tipos de las instalaciones.

A fin de completar el conjunto de datos sobre costos, deben darse también los costes específicos de producción y las clases de amortización, incluido capital invertido. Si ahora el departamento de personal da las cargas correspondientes a los lugares de gastos (tarjetas perforadas), pueden informar los programas de evaluación de ese conjunto de datos sobre los costos de una instalación o bien de un lugar de gastos. Simultáneamente, se calculan las ganancias o pérdidas acumuladas en caso de variaciones de producción y quedan a disposición de la dirección a fin de tomar decisiones. Para la realización de este sector parcial del sistema de información, se debe investigar cuidadosamente sobre qué calculadoras deben realizar los programas individuales (calculadora de procesos o sistema comercial). Sin embargo, no se ha discutido aún la exigencia de presentar todos los datos 
en formato manejable y la de utilizar, en tanto sea posible, los mismos medios de acumulación (por ejemplo placas intercambiables). La distribución de las funciones de la calculadora depende grandemente de la organización de la empresa, de modo que el pretender aquí una discusión sobre los detalles nos llevaría demasiado lejos.

\subsubsection{Análisis y planificación}

Las siguientes tareas de un sistema general de información en el sector del cálculo de costos no han de ser tratados aquí, pues son resueltos actualmente del modo convencional o con las calculadoras comerciales de las empresas:

- planificación de los medios de liquidez;

- predicción de presupuestos;

- planificación de los repuestos de las instalaciones;

- análisis de los costos y de las ganancias.

\section{EXIGENCIAS PARA LA CALCULADORA}

Como portadora del sistema de información, se ha supuesto una calculadora de procesos de modo que quedan fijadas implícitamente determinadas exigencias:

Por causas de la seguridad en el servicio de los programas de regulación, debe permanecer automáticamente en marcha tras un fallo en el suministro de fluido eléctrico. Durante la interrupción de la corriente deben permanecer los elementos de ajuste sobre el valor último útil o marchar a su posición final de seguridad. Debido a que todos los circuitos de regulación rápida (ciclo de exploración de 1 a 5 segundos) son accionados, incluso en servicio de calculadora, mediante reguladores analógicos, no es muy crítico el fallo de la calculadora para las regulaciones lentas. Entonces puede continuar la marcha el personal de servicio, de la forma acostumbrada en servicio manual.

Como consecuencia del servicio con tiempo real, debe poseer la calculadora una vigilancia interna de tiempo que persigue el proceso ordenado del trabajo de los programas. Por motivo del gran número de los datos de proceso y de los programas de regulación, es necesario su almacenamiento en unidades externas. De ello resulta, que la velocidad de trabajo del sistema depende menos del tiempo del ciclo de la unidad central que de los tiempos de ataque a los almacenes de placas y del tipo de transmisión al acumulador central.

Se hace posible elevar la velocidad total de trabajo, cuando los datos y los programas están dispuestos en placas separadas de modo que los brazos de agarre han de recorrer caminos menores en relación al sector elaborado.

El tamaño del acumulador central debe estar comprendido entre 24 y $32 \mathrm{~K}$ palabras a causa de la cantidad de programas distintos a los del proceso.

En él son intercambiables de 7 a $15 \mathrm{~K}$ palabras de memoria por los sectores de programa, sobre las placas o galletas. 
Las memorias externas se disponen del siguiente modo:

- 1: programa de mando (ejecutivo) y datos referentes al programa;

- 2: programas de procesos;

- 3: programas para la acumulación de información y condensación;

- 4: programas para el análisis de sistemas y optimación (intercambiables);

- 5: programas de mantenimiento, almacén de piezas de repuesto (intercambiables);

- 6: facturación y expedición.

Los tres acumuladores últimamente enumerados pueden unirse también en grandes fábricas a un sistema de elaboración de datos para fines comerciales. Entonces, la transmisión de datos al calculador de procesos tiene lugar a velocidad relativamente lenta, mediante transmisión de datos a distancia. Este segundo sistema debiera, sin embargo, poder programarse también en FORTRAN y ser compatible con la calculadora de procesos en relación al portador de datos.

Por el contrario, para pequeñas fábricas, se recomienda aplicar una de las placas intercambiables, la 4 o bien la 5, para los trabajos comerciales, en tanto su volumen puede ser acomodado aún en el tiempo libre del calculador de procesos.

Debido a que el sistema del calculador debe elaborar tanto programas de proceso como de no proceso, se hace necesaria su distribución en niveles de interrupción de diversa prioridad.

El cambio de los sectores variables del acumulador central con la placa necesita determinados tiempos para coger y transmitir los datos.

Por lo tanto, se ha de elegir de forma tal el ciclo del programa de proceso que el tiempo libre para el programa de no proceso importe por lo menos, en cada ciclo, de 3 a 5 segundos. De otro modo resulta demasiado desfavorable la relación de intercambio al tiempo de trabajo.

Otro progreso de los códigos de calculadoras son los sistemas de interpretación, que están actualmente a disposición tanto para el cálculo directo digital como para las funciones puras de vigilancia.

La ventaja principal reside en su corto tiempo de programación, que consiste casi únicamente en el relleno de formularios y su perforado en tarjetas. Con ellas pueden ser representadas todas las funciones necesarias del proceso; pero, sin embargo, no es posible efectuar las rutinas de evaluación y condensación descritas en este sistema de información, como, por ejemplo, para las tareas de entretenimiento. Pero pueden ser programadas en FORTRAN, en donde puede ser aprovechada ventajosamente la estructura de tablas del sistema de interpretación, pero con otras funciones. Si se decide por una programación unitaria en FORTRAN, incluso para las funciones de regulación, es posible hacerlo sin dificultades, como muestran numerosas instalaciones con pleno éxito en la industria americana del cemento. Allí están inscritos en FORTRAN aproximadamente el $95 \%$ de los programas y el resto en lenguaje simbólico. 


\section{NECESIDADES DE TIEMPO Y PERSONAL}

El plan de tiempos para la creación del sistema de información es el siguiente:

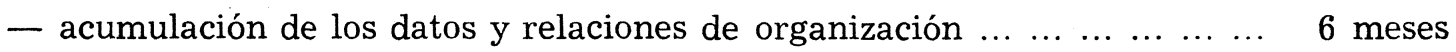

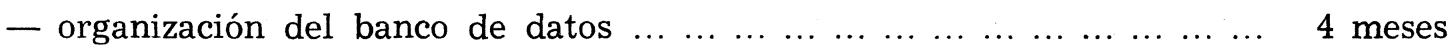

— desarrollo y realización de los programas de proceso $\ldots \ldots \ldots \ldots \ldots \ldots \ldots$. . . . . 3 meses

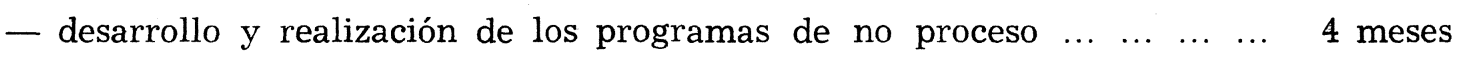

$\begin{array}{lllllllllllllll}- & \text { acomodación de } \operatorname{los} \operatorname{programas} \\ \ldots & \ldots & \ldots & \ldots & \ldots & \ldots & \ldots & \ldots & \ldots & \ldots & \ldots & \ldots & \ldots & \ldots & 4 \\ \text { meses }\end{array}$

Para la creación del sistema se hace necesario el siguiente personal:

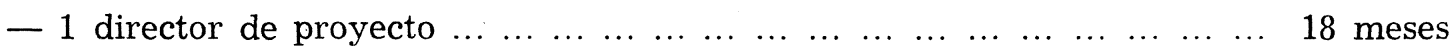

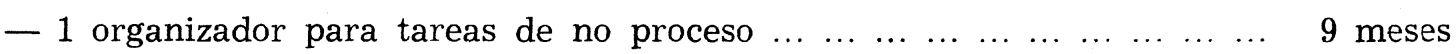

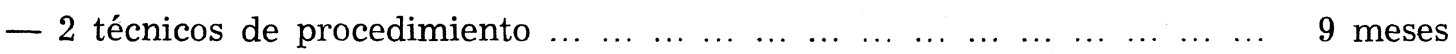

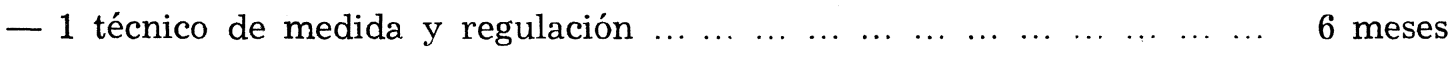

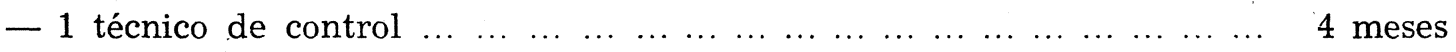

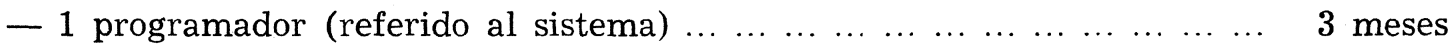

$\begin{array}{lllllllllllllllll}-1 & \text { programador }(e s t a d i ́ s t i c o) & \ldots & \ldots & \ldots & \ldots & \ldots & \ldots & \ldots & \ldots & \ldots & \ldots & \ldots & \ldots & \ldots & \ldots & 4 \\ \text { meses }\end{array}$

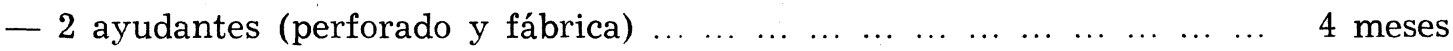

Condición precisa para conseguir estos tiempos es la seguridad de todos los colaboradores, tanto con las instalaciones y procedimientos como con la clave de programación FORTRAN. El personal aquí detallado, de aproximadamente 5 hombres-año, no se puede reducir esencialmente cuando en lugar de efectuar la programación en FORTRAN es utilizado un sistema de interpretación. De todos modos, el costo se puede reducir algo, con la instalación de un segundo sistema similar para una estructura semejante de la fábrica. 